Résumés des conférences et travaux

\title{
Langue et littérature latines du Moyen Âge
}

Conférences de l'année 2011-2012

Anne-Marie Turcan-Verkerk

\section{OpenEdition}

$\checkmark$ Journals

Édition électronique

URL : https://journals.openedition.org/ashp/1479

DOI : 10.4000/ashp.1479

ISSN : 1969-6310

Éditeur

Publications de l'École Pratique des Hautes Études

\section{Édition imprimée}

Date de publication : 1 septembre 2013

Pagination : 102-107

ISSN : 0766-0677

\section{Référence électronique}

Anne-Marie Turcan-Verkerk, "Langue et littérature latines du Moyen Âge », Annuaire de l'École pratique des hautes études (EPHE), Section des sciences historiques et philologiques [En ligne], 144 | 2013, mis en ligne le 23 octobre 2014, consulté le 05 novembre 2021. URL : http://journals.openedition.org/ashp/ 1479 ; DOI : https://doi.org/10.4000/ashp.1479 


\title{
LANGUE ET LITTÉRATURE LATINES DU MOYEN ÂGE
}

\author{
Directeur d'études : M ${ }^{\text {me }}$ Anne-Marie TurCAN-VERKERK
}

Programme de l'année 2011-2012 : I. La tradition des traités de versification latine aux XII et XIII ${ }^{e}$ siècles. - II. La bibliothèque médiévale et la mémoire des défunts.

\section{La tradition des traités de versification latine aux XII et XIII ${ }^{e}$ siècles}

Les traités de prosodie et de versification tant métrique que rythmique jouent un rôle important dans le classement des manuscrits du Liber artis omnigenum dictaminum de maître Bernard dit « de Bologne », présenté en 2010-2011. L'année 20112012 a été consacrée à l'analyse de leur genèse, de leur diffusion et de leurs avatars jusqu'au XIV ${ }^{\mathrm{e}}$ siècle.

Les conditionnements intellectuels dus à la bibliographie antérieure ont entravé la compréhension de l'ars dictandi comme un tout comprenant à la fois l'art d'écrire en prose et l'art d'écrire en vers, et par conséquent empêché une série de découvertes, tant sur la genèse d'arts de versification plus modestes que les traités édités par Edmond Faral en 1924, que sur leur datation et leur localisation. Le travail mené au cours de la conférence a pour objectif de redonner leur place à ces textes négligés et souvent ignorés.

Nous nous sommes tout d'abord attachés à l'étude du premier traité de prosodie et de versification métrique produit par maître Bernard au début de sa carrière, vers 1140, dont le témoin le plus ancien (et longtemps le seul repéré) est le manuscrit München, BSB, lat. 14784 f. 38-43v. Ce premier essai du maître en dictamen vise à fournir aux élèves un compendium minimal, composé progressivement par l'agrégation de sources nouvelles. Le manuel, qui nous a été transmis comme un texte continu sans ruptures apparentes dans la copie, présente des redites, des contradictions, des ruptures. L'analyse de ce qui pourrait apparaître comme des accidents textuels, en réalité des micro-fractures et des doublons, montre que chacun d'entre eux correspond en fait au passage à une source nouvelle. On peut donc comprendre comment Bernard a travaillé, et dans quel ordre il a dépouillé les ouvrages antérieurs : il a commencé par l'Ars maior de Donat et le commentaire de Donat par Servius, puis il a commencé à dépouiller le De arte metrica de Bède, et enfin il a découvert Priscien. Le De finalibus de Servius (joint à Bède, qui redevient vers la fin sa source principale) pousse Bernard à inventer la méthode de la diminutio permettant de retrouver la longueur native des syllabes à partir de l'accent tonique.

Bernard a-t-il entièrement dépouillé Priscien pour produire son propre manuel? A-t-il utilisé des excerpta? Les excerpta de Priscien sont encore trop mal connus pour que l'on puisse le déterminer. Le ms. lat. 17209 de la BSB de Munich, qui transmet aux f. 64-65 ce même traité de prosodie, suivi au dernier feuillet (f. 65-65v), comme dans le clm 14784, du traité de Bernard sur l'hexamètre rimé, transmet également des 
excerpta de Priscien « et d'autres maîtres » (f. 29-53[?]), dans lesquels on note avec intérêt le mot maneries (dans l'expression dictionum maneries), habituellement signature de maître Bernard. L'incipit rappelle d'autres incipit bernardins (Quoniam de partibus orationis quorumdam sociorum nostrorum rogatu compendiosum tractatum ad declinationis seriem ex Prisciani et aliorum magistrorum libris colligere breviter proposuimus...), et l'on trouve ici le même souci de formuler des règles générales (du type : Omnia..., Omnes...) et de donner des méthodes infaillibles. Ces extraits ne concernent pas la prosodie, mais ils peuvent procéder eux aussi d'une lecture complète de Priscien : ils sont entièrement à étudier et pourraient enrichir le dossier de Bernard, maître de grammaire avant tout. Cependant l'existence d'un traité de prosodie attribué à Hraban Maur, et fabriqué de la même façon à partir d'un dépouillement de Priscien, pose un certain nombre de problèmes. Ce manuel qui se présente comme des extraits de Priscien ne nous est connu que par son édition princeps, et il offre des parentés troublantes avec les nouveaux traités des $\mathrm{XI}^{\mathrm{e}}$ et $\mathrm{XII}^{\mathrm{e}}$ siècles. S'agit-il vraiment d'un texte carolingien, que Bernard aurait pu connaître (mais comment s'il n'a pas été diffusé?), ou s'agit-il au contraire d'une variante tardive des traités du Moyen Âge central? En l'absence d'édition critique de l'ensemble de cette littérature, il est impossible de trancher : le traité présente de très nombreuses parentés avec les travaux bernardins - mais l'on a parfois le sentiment que Bernard a pu connaître des travaux d'auteurs carolingiens d'outre-monts, comme le suggère une citation de Priscien, dans laquelle la séparation aberrante des mots grecs ne semble pouvoir s'expliquer que par la ponctuation ajoutée au manuscrit Leiden UB BPL 67 par Jean Scot et son associé.

Ainsi, ce que nous avons dans les clm 14784 et 17209 est la copie d'une suite de fiches plus ou moins mises en forme mais qui n'ont pas encore trouvé leur place dans un plan cohérent. Ce cours de prosodie pour débutants a été professé, puisque l'on trouve la trace de certains de ses éléments les plus personnels (en l'absence de source commune repérable) chez Paul le Camaldule et chez Uguccio de Pise.

Ce premier essai a certainement rendu des services aux élèves de Bernard, qui lui ont demandé de rédiger un véritable manuel, comme en atteste la préface en trois distiques élégiaques du traité augmenté, transmis à ce jour dans sa version initiale par le seul manuscrit Erfurt, UB, CA $8^{\circ} 16$ f. $52-58 v^{1}$. Nous avons mis l'accent sur le saut qualitatif qui sépare cette nouvelle version du premier essai. Le plan a été largement repensé, ce qui supprime les redites comme les contradictions. Cependant, il n'est pas encore entièrement logique, et le texte conserve, ici ou là, des traces de notes additionnelles, d'éléments en attente d'intégration; une partie de ces imperfections sont corrigées dans un état de peu postérieur, dont témoigne Admont, SB, 759 f. 1v-21v. L'ensemble du traité fait l'objet d'une normalisation, qui gomme en particulier les traces de la pédagogie personnelle de Bernard, présentes dans la version «brute». L'événement majeur qui s'est produit entre la première version, associée aux Rationes

1. Cette préface métrique ayant fait croire à un catalogueur du $\mathrm{Xv}^{\mathrm{e}}$ siècle que maître Bernard était Bernard Silvestre, la présentation de ce texte a été l'occasion de faire le point sur la confusion des deux Bernard, qui a perduré. Bernard Silvestre n'a sans doute pas fait autre chose, dans le domaine du dictamen, qu'enseigner à partir des manuels de maître Bernard, alors introduits en France et retravaillés à Tours (comme le démontrera la conférence de 2012-2013). 
dictandi dans le clm 14784, et ce second traité est cependant d'une autre nature : il s'agit de la découverte d'une source nouvelle, les traités de prosodie d'Albéric du Mont-Cassin. Bernard, au moment où il avait constitué le dossier d'œuvres complètes d'Albéric que nous transmet le clm 14784, ne les connaissait pas encore.

À l'occasion de cette découverte par Bernard, entre 1140 et 1145, de la méthode dite « $\mathrm{A}$ ante $\mathrm{B}$ », nous nous sommes interrogés sur la genèse de cette façon de déterminer la longueur native des syllabes, promise à un très grand succès, grâce en par-

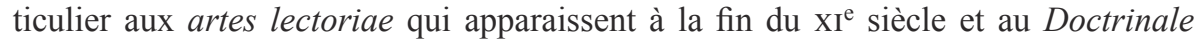
d'Alexandre de Villedieu quelque cent ans plus tard. Les deux premiers auteurs qui la mettent en œuvre au $\mathrm{XI}^{\mathrm{e}}$ siècle sont Tebald de Plaisance, dont la datation exacte n'est pas connue, et Albéric du Mont-Cassin. Tebald, dont le traité en vers a connu une importante diffusion et peut-être plusieurs versions, est aujourd'hui inédit et de ce fait difficilement exploitable. Les traités en prose d'Albéric, eux, sont transmis dans leur version probablement originale par un manuscrit unique, Vaticano, BAV, Ottob. lat. 1354, mais ils ont connu divers avatars, dont une version transmise par un manuscrit de l'Ambrosienne de Milan, M 79 sup. f. 250va-f. 252b (foliotation manuscrite). Maître Bernard en a utilisé un état sans doute intermédiaire. Les traités d'Albéric remontent, avec l'ensemble des manuels de l'Ottob. lat. 1354, aux années de son enseignement qui ont précédé sa « conversion » à l'ars dictandi, avant 1079 donc. La conférence a mis l'accent sur la parenté de méthode entre ces traités alphabétiques de prosodie et l'Elementarium de Papias, écrit à l'époque où Albéric faisait ses études : une raison peut-être qui, ajoutée à certaines parentés textuelles étroites entre l'Elementarium et le Breviarium d'Albéric (en particulier sur les privilèges), pourrait s'ajouter aux arguments de Violetta De Angelis laissant penser que Papias a travaillé et enseigné au Mont-Cassin. L'utilisation de ces véritables dictionnaires prosodiques comme glossaires à l'usage des versificateurs est d'ailleurs attestée par le manuscrit principal de Bernard, Savignano sul Rubicone, Accad. dei Filopatridi, 45, qui intègre de première main et à l'encre rouge des gloses proposant des synonymes ou des explications.

La version finale du traité de prosodie, intégrée au Liber artis omnigenum dictaminum, témoigne d'une très bonne rationalisation du plan, qui traite des syllabes dans leur ordre d'apparition (initiales, médianes, finales) et évite les redites, et d'un effort général de normalisation. Datable dans son état achevé de 1145, comme l'ensemble de la summa, ce traité réserve une surprise : une citation, déjà sous le nom d'Ovide, du premier vers du De pulice, que l'on ne date habituellement que de l'extrême fin $\mathrm{du} \mathrm{XII}{ }^{\mathrm{e}}$ siècle au plus tôt. Cette découverte, déjà signalée dans le rapport 2008-2009, remet en cause à la fois la datation et la localisation de ce texte, qui pourrait être d'origine italienne. Elle invite aussi à lire d'un autre œil l'histoire littéraire des « petites bêtes " : en effet, pour des raisons de chronologie évidentes, on s'interdisait jusqu'à ce jour d'établir des parentés, pourtant très tentantes, entre le De pulice et les Pulicis et musce iurgia de Guillaume de Blois, écrits vers 1170. Mais Guillaume était le frère de Pierre de Blois, qui, vers 1160, avait fait ses études de droit et de dictamen à Bologne, certainement auprès de Bernard - s'il vivait encore - et au moins de Guido son élève. Pierre, que la poésie légère n'effarouchait pas dans sa jeunesse, pourrait avoir transmis aux milieux tourangeau et plus largement ligérien un pseudo-Ovide connu à Bologne au moins depuis 15 ans. 
L'année s'est terminée sur la présentation des avatars médiévaux du traité de Bernard sur les hexamètres rimés, qui a connu un grand succès dans des maisons cisterciennes de l'espace germanique, sans doute à la suite de la constitution, à l'abbaye cistercienne de Viktring, d'un recueil des traités de versification de Bernard visant à expurger l'ars dictandi de toute trace de poésie. Succès paradoxal, mais effectif. Endehors de ces réseaux cisterciens, l'accent a été mis plus particulièrement sur une récriture du traité attribuable à Jean de Garlande, travail lié sans doute à l'exploitation des traités de versification de Bernard, tout spécialement de versification rythmique, pour la Parisiana poetria.

\section{La bibliothèque médiévale et la mémoire des défunts}

Si le sens antique de bibliotheca n'a cessé d'être connu pendant tout le Moyen Âge par les sources littéraires et encyclopédiques, en particulier grâce à Isidore, si les auteurs médiévaux ont utilisé régulièrement le terme dans cette acception, les documents issus de la plume des armarii l'ignorent presque totalement jusqu'à la fin du $\mathrm{XI}^{\mathrm{e}}$ siècle. Dans le monde de la bibliothéconomie du haut Moyen Âge, la bibliotheca est exclusivement la Bible, collection de textes et corpus unifié par excellence. Le bibliothecarius quant à lui est essentiellement, à l'image de la fonction qui reparaît à Rome au $\mathrm{IX}^{\mathrm{e}}$ siècle, un archiviste. Au cours du XII ${ }^{\mathrm{e}}$ siècle (apparemment) commence à s'imposer, pour la Bible, le terme biblia. La concomitance du passage de bibliotheca à biblia pour désigner la Bible et du changement de sens de bibliotheca sous la plume des « professionnels » de la bibliothèque n'a jamais suscité, semble-t-il, de réflexion. La conférence s'est donc interrogée sur les modalités et les raisons de ce transfert de sens qui s'opère à la fin $\mathrm{du} \mathrm{XI}^{\mathrm{e}}$ siècle. Ce transfert est exactement contemporain d'un autre : catalogus, traditionnellement utilisé pour les listes de noms d'hommes ou plus généralement pour les listes normatives, telles les listes de vices et de vertus, ne devient relativement fréquent pour désigner les listes de livres qu'à partir de la fin du $\mathrm{XI}^{\mathrm{e}}$ siècle. Pourquoi, et pourquoi ces deux transferts ont-ils été contemporains?

Si le passage de bibliotheca à biblia peut éventuellement s'expliquer par des changements codicologiques (nombre de volumes occupés par la copie de la Bible plus restreint, voire réduit à un, Bible de plus en plus portable, de moins en moins liée à un lieu de conservation), la raison fondamentale n'est certainement pas là. Nous avons cherché une raison plus profonde dans les affinités, sensibles surtout à partir $\mathrm{du} \mathrm{XI}{ }^{\mathrm{e}}$ siècle dans le monde clunisien, entre structuration de la mémoire des morts et structuration de la mémoire des livres : c'est ici que l'analyse du mot catalogus prend son sens. C'est dans le creuset clunisien que se structurent hiérarchiquement les catalogi defunctorum, comme en atteste le Liber tramitis, ainsi que plusieurs libri viventium, qui utilisent pour cela, surtout en domaine germanique, la forme mémorielle des arcades. Les listes de noms organisées dans un ordre hiérarchique descendant et éventuellement disposées dans des structures à arcades représentant l'ecclesia ou la Jérusalem céleste fournissent à certains catalogueurs, comme celui d'Arnstein ou celui de Prüfening, le cadre mémoriel permettant de transmettre la liste des livres, d'abord liste d'auteurs, à la postérité : elles font de l'inventaire des livres un véritable liber viventium des auteurs, un catalogus librorum. La hiérarchie des auteurs est pensée 
d'abord comme une hiérarchie de défunts dont on fait mémoire, ce qui explique bien des traits de la bibliothéconomie ancienne, qui met par exemple l'accent sur le nomen du livre en négligeant la catégorie du titulus, qui désigne volontiers le livre par le nom de l'auteur suivi d'un titre au génitif, qui fait des auteurs des fondateurs avant d'en faire des écrivains. Cluny, qui organise la mémoire des défunts, fusionne plus que tout autre dans la fonction de l'armarius le soin de l'écrit (archives et livres) et la memoria des défunts, comme l'ont montré les travaux de Jean-Loup Lemaitre, mais même en dehors de l'ecclesia cluniacensis les inventaires sont transmis plus souvent que l'on ne l'imagine dans un contexte nécrologique. Cluny organise aussi, au même moment, la bibliothèque, avec son grand catalogue structuré, monumental, et exposé en un lieu dédié au rangement des livres : cet espace consacré à la bibliothèque est mentionné dans les coutumes de Guillaume de Hirsau, qui emploie enfin le mot bibliotheca, en expliquant qu'il désigne la même chose qu'armarium; le grand catalogue de la bibliothèque, certainement à peu près contemporain, utilise un terme ecclésial et royal pour désigner ce même espace où l'on n'a le droit ni de lire, ni d'écrire, ni de parler : aula.

Qui dit hiérarchie dit ordre, mais qu'est-ce que l'ordre, et par quoi est-il fondé? Bien que cette question soit, de toutes, la plus fondamentale, elle a été traitée rapidement en fin d'année et ne peut être ici que drastiquement résumée. L'analyse d'ordo dans les inventaires de livres n'apporte pas de réponse, car le sens le plus courant est celui d'étagère; dans le meilleur des cas, ordo désigne la succession physique sur les rayons, sans notion de classement, ou l'ensemble des livres entendu comme une catégorie d'objets. Ce qui fonde la hiérarchie des auteurs dans les inventaires médiévaux, c'est leur proximité, chronologique mais surtout qualitative, avec l'Écriture sainte. Celle-ci est le point de départ et d'arrivée du processus de la connaissance.

Origine de la connaissance, elle est en tête des catalogues, suivie immédiatement de ses interprètes, le choix du premier d'entre eux revêtant une signification particulière. À Augustin, Cluny préfère Grégoire à la fin $\mathrm{du} \mathrm{XI}^{\mathrm{e}}$ siècle, favorisant ainsi divers processus d'identification; Cluny transmet la structure de son catalogue aux maisons clunisiennes et surtout néo-clunisiennes, transformant ainsi un choix spirituel et politique en progrès bibliothéconomique : l'ordre se traduit statiquement par la structure du catalogue, désormais exportable.

L'Écriture est aussi le point d'aboutissement du processus de formation, qui vise à rendre l'homme capable de contempler Dieu à travers la rumination de l'Écriture. Pour cela, l'homme doit se former selon un programme qui le mène de livre en livre (programme décrit par le livre I des Institutiones de Cassiodore), grâce à des outils (livre II des Institutiones). La formation est ainsi un ordo dans le sens que lui donne Augustin dans le De ordine, un ordre dynamique, que la plupart des catalogues médiévaux structurés expriment comme Cassiodore par la bipartition lettres divines/lettres profanes, et parfois en respectant la succession cassiodorienne des textes.

Cet ordo d'ascendance néo-platonicienne, qui fonde la bibliothèque et le catalogue qui la décrit, a été plusieurs fois représenté par la métaphore de la maison spirituelle, par exemple par Hugues de Saint-Victor (chez qui elle entretient des affinités avec l'idée de la construction historique), ou, de façon plus aboutie, par le catalogueur du $X^{e}{ }^{e}$ siècle de la Chartreuse d'Erfurt; les Pères sont les murs porteurs d'un édifice dont le faîte est la Bible : entrer et progresser dans la maison, c'est s'approcher de son 
sommet. Dans cette maison, on retrouve une structure mémorielle bien connue depuis l'Antiquité. Le lien est fondamental entre l'architecture de la mémoire collective - la bibliothèque - et la construction de soi à travers le cheminement de lecture en lecture. Voilà qui explique, sans doute, que les catalogues médiévaux cherchent à structurer les lettres divines mais ne se soucient jamais de structurer les lettres profanes : structurer les simples outils n'a pas de sens. La bibliothèque et son catalogue fixant la mémoire ordonnée des auteurs assurent la continuité permettant à chaque individu de cheminer à son tour vers la connaissance et d'ajouter parfois son nom - sa pièce - à la construction collective. La bibliothèque, communauté des vivants, mémoire des morts sans laquelle les vivants ne peuvent se construire. 